\title{
Studies on the interaction of rheumatoid factor with monosodium urate crystals and case report of coexistent tophaceous gout and rheumatoid arthritis
}

\author{
T P GORDON, M J AHERN, C REID, AND P J ROBERTS-THOMSON
}

From the Department of Clinical Immunology, Flinders Medical Centre, Bedford Park, South Australia 5042

SUMMARY Gout and classical rheumatoid arthritis rarely coexist. We report a patient with strong evidence for both these diseases. Possible reasons for the negative correlation between these $\omega_{\infty}^{\omega}$ diseases are summarised. One hypothesis suggests inhibition of surface activity of monosodium urate crystals (MSU) by binding of rheumatoid factor (RF). This was studied with a purified ${ }^{\circ}$ monoclonal rheumatoid factor (mRF) with specificity for IgG. The mRF bound preferentially to $\bar{c}$ MSU coated with IgG in contrast with the IgM control. Inhibition of the neutrophil $\frac{\sigma}{\sigma}$ chemiluminescence (CL) response to IgG-coated MSU was observed at concentrations of $\mathrm{mRF}_{\overrightarrow{0}}$ that had no effect on the CL response to uncoated crystals. Neutrophil activation was not altered $\infty$ by coating crystals with an IgM control at the same concentration. These data suggest that RF may bind to antigenic determinants on exposed Fc of adsorbed IgG and block the interaction of crystal-bound IgG with Fc receptors. Although crystal coating by RF may modify the expression of gouty arthritis, it is unlikely to be the sole explanation for the dissociation between gout and RA.

The negative correlation between gout and rheumatoid arthritis (RA) is widely accepted, but the reason(s) are unclear. ${ }^{1}$ Since synovial thickening, ${ }^{2}$ symmetrical joint involvement, ${ }^{3}$ a low titre rheumatoid factor $^{4}$ (RF), joint erosions, ${ }^{5}$ and similar histopathological changes in the synovial membrane $^{6}$ can occur in both conditions, proof of the coexistence of gout and RA requires the demonstration of monosodium urate crystals (MSU), a high titre RF, and histological evidence of a rheumatoid nodule in an alcohol-fixed specimen. ${ }^{7-9}$

Possible reasons for the mutual exclusion between these diseases have been reviewed elsewhere. ${ }^{110}$ and include an anti-inflammatory or immunosuppressive effect of hyperuricaemia, ${ }^{11-14}$ inhibition of crystal deposition by possible connective tissue alterations in RA, ${ }^{15}$ diagnostic difficulty, synovial hypocomplementaemia, ${ }^{16}$ and impaired phagocytic function of neutrophils in rheumatoid joint fluid. ${ }^{1617}$ It has also been proposed that coating of MSU by RF may inhibit the activation of cellular or fluid-phase mediators of inflammation by the crystal surface. ${ }^{13} 18$

- Accepted for publication 7 December 1984. Correspondence to Dr T P Gordon.
We report the fourth recorded case of coexistent gout with MSU and RA with high-titre RF and a biopsy-proved rheumatoid nodule. With in-vitro techniques and a purified monoclonal rheumatoid: factor (mRF) with specificity for IgG we have tested3. the hypothesis that binding of RF to adsorbed $\mathrm{IgG}$ on the MSU surface might modify gouty inflammation in patients with RA.

\section{Case report}

A 60-year-old man had a history from 1958 of episodic acute attacks involving the feet, ankles, andr wrists, which responded to treatment with colchicinen or indomethacin. In 1960 he developed persistent ${ }_{\sigma}^{\omega}$ pain and stiffness of multiple joints and synovitis of the proximal interphalangeal (PIP), metacarpopha-c langeal (MCP), wrist, and elbow joints bilaterallyo and symmetrically and a positive RF titre of $1 / 2048^{-}$ (Rose-Waaler). In 1968 needle aspiration of nodulesoover the pinnae of the ears, olecranon bursa, and产 right tibia showed MSU by compensated polarised light microscopy (CPLM). The serum uric acid levelo was $0.5 \mathrm{mmol} / 1$ (range $0.15-0.42 \mathrm{mmol} / \mathrm{l}$ ). Allopur $>$ inol $300 \mathrm{mg}$ daily was started but ceased in 19798 
because of a rash. Over this period he experienced no further 'gout-like' episodes, but the symmetrical polyarthritis persisted.

In 1981 bilateral synovial thickening of the PIP, MCP, wrist, and elbow joints, large knee effusions, and flexor tenosynovitis of the fingers were all noted. Tophi from which MSU crystals were recovered were present over the olecranon and dorsum of the right wrist. Investigations showed a haemoglobin of $11.7 \mathrm{~g} / \mathrm{dl}$, ESR $60 \mathrm{~mm} / \mathrm{h}$, serum uric acid 0.74 $\mathrm{mmol} / \mathrm{l}, \mathrm{RF}$ titre $1 / 2048$. Joint fluid of low viscosity was aspirated from the right knee and showed a white blood cell count of $12 \times 10^{9} / 1$ with $89 \%$ neutrophils and $11 \%$ mononuclear leucocytes, and a RF titre of $1 / 16384$. No urate crystals were detected in knee joint fluid on this or subsequent aspirations. Radiographs of the hand showed erosive changes in the radiocarpal, intercarpal, and MCP joints. Erosions were present in the first metatarsophalangeal joints, right interphalangeal joint of the big toe, and the heads of the metatarsal bones. During admission both intra- and extracellular urate crystals were recovered from an acutely inflamed ankle joint. After resolution of the attack allopurinol 300 $\mathrm{mg} /$ day was reinstituted.

The patient was readmitted for bilateral knee joint replacement in June 1984. Tophi in the pinnae of the ears and over the olecranon and right wrist had disappeared. Serum uric acid was $0.37 \mathrm{mmol} / \mathrm{l}$ and RF titre 1/256. Excisional biopsy of a nodule over the extensor aspect of the left forearm, fixed in absolute alcohol, showed several rheumatoid nodules each consisting of a central area of necrosis surrounded by palisading mononuclear cells (Fig. 1). Examination of the unstained, alcohol-fixed specimens by CPLM was negative for MSU. Syno-

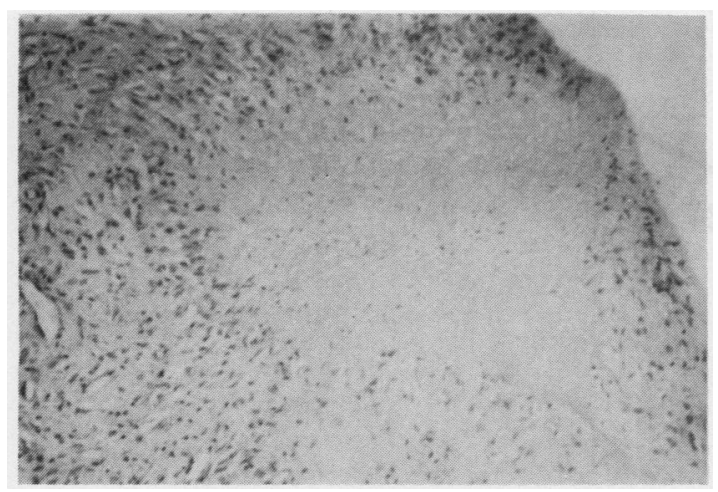

Fig. 1 Haematoxylin and eosin stain of a rheumatoid nodule from the extensor aspect of the forearm showing central area of necrosis surrounded by palisading mononuclear cells. No urate crystals were seen in the unstained alcohol-fixed specimen. $(\times 100)$. vial membrane from the knee joint showed synovial lining cell hyperplasia and infiltration with mononuclear and polymorphonuclear leucocytes, changes compatible with but not pathognomonic of RA. ${ }^{6}$ No urate crystals were detected in the synovial specimens. Treatment was continued with indomethacin and allopurinol and he was started on Dpenicillamine.

\section{Materials and methods}

NEUTROPHILS

Human peripheral neutrophils were obtained from heparinised blood by Hypaque-Ficoll separation followed by dextran sedimentation as described previously. ${ }^{19}$ Contaminating red cells were lysed by a five minute incubation in Gey's solution and the preparation adjusted to $5 \times 10^{9}$ neutrophils/l in Eagle's minimal essential medium (MEM, Flow Laboratories) containing $1 \%$ fetal calf serum.

\section{CRYSTALS}

MSU crystals were prepared under sterile conditions by the method of Denko and Whitehouse ${ }^{20}$ and the supersaturated solution autoclaved before crystallisation. Crystals were washed twice in distilled water, dried under UV light for 72 hours, and resuspended in urate-saturated Dulbecco's phosphate-buffered saline (PBS) at a concentration of $10 \mathrm{~g} / \mathrm{l}$. After sonication at 1500 cycles per second for one minute a stock suspension with crystals ranging from 1 to $20 \mu \mathrm{m}$ in length was stored at $4^{\circ} \mathrm{C}$.

Crystals were coated with IgG by incubation with Cohn fraction 11 (CSL) in a ratio of $10 \mathrm{mg}$ crystals per $1 \mathrm{ml}$ of protein solution $(5 \mathrm{~g} / \mathrm{l})$ for 30 minutes at room temperature, then washed three times in urate-saturated PBS, and resuspended at a concentration of $10 \mathrm{~g} / \mathrm{l}$. When comparisons were made between IgG-coated and uncoated crystals, both preparations were washed equally. ${ }^{21}$

PURIFICATION AND RADIOLABELLING OF MRF AND IgM

The $\mathrm{mRF}$ was purified from the serum cryoglobulin of a patient with a lymphoproliferative disorder as described previously $(\operatorname{mRF}(A)) .^{22}$

The IgM control was prepared from the serum of a patient with Waldenström's macroglobulinaemia whose IgM level was $55 \mathrm{~g} / \mathrm{l}, \operatorname{IgG} 0.35 \mathrm{~g} / \mathrm{l}$, and IgA $<0.07 \mathrm{~g} / \mathrm{l}$. The serum was fractionated on a Sepharose $6 \mathrm{~B}$ column, and the fractions containing IgM were pooled and concentrated in an Amicon concentration unit. The final concentrations of the $\mathrm{mRF}$ and IgM were determined spectrophotometrically; both were adjusted to $0.5 \mathrm{~g} / \mathrm{l}$ in PBS and stored at $-80^{\circ} \mathrm{C}$ before use. Aliquots of $\mathrm{mRF}$ and 
IgM were radiolabelled with ${ }^{125}$ I by a modification of the lactoperoxidase method with $500 \mu \mathrm{g}$ protein and $200 \mathrm{mCi}^{125} \mathrm{I}^{23}$ Non-protein iodine was removed by passing through a Sephadex G25 column. The percentage precipitations of ${ }^{125} \mathrm{I}-\mathrm{mRF}$ and ${ }^{125} \mathrm{I}-\mathrm{IgM}$ in $10 \%$ trichloroacetic acid were greater than $95 \%$.

BINDING OF ${ }^{125}$ I-mRF AND ${ }^{125}$ I-IgM TO MSU $100 \mu \mathrm{l}$ of tracer amounts of ${ }^{125}$ I-labelled protein were incubated with MSU for 60 minutes at room temperature with frequent vortexing. Unbound protein was removed by a modification of the method of Terkeltaub et al. ${ }^{24}$ Crystals were washed through $1.0 \mathrm{ml}$ of $40 \%$ sucrose in urate-saturated PBS and then washed once in urate-saturated PBS. The washed crystal pellet was resuspended in $0.5 \mathrm{ml}$ PBS and counted separately from the supernatant and tube. The percentage binding of ${ }^{125} \mathrm{I}-\mathrm{mRF}$ and ${ }^{125} \mathrm{I}-\mathrm{IgM}$ to MSU was calculated from the formula cpm pellet/cpm supernatant $+\mathrm{cpm}$ pellet $+\mathrm{cpm}$ tube $\times 100 \%$.

LUMINOL

A stock solution of 0.056 M 3-aminophthal- hydrazide (Koch Light Laboratories) in dimethyl $\frac{\mathrm{D}}{\mathrm{N}}$. sulphoxide was diluted 1:40 in MEM before use. $\stackrel{\vec{*}}{\vec{*}}$

CHEMILUMINESCENCE (CL) ASSAY

In order to measure the interaction between MSU and neutrophils a $\mathrm{CL}$ assay was used. $\mathrm{CL}$ is an integral part of the respiratory burst of stimulated neutrophils and an indirect measure of oxygen free radical generation. Thus CL reflects early membrane activation of the neutrophil. ${ }^{25}$ The CL response of human neutrophils was measured as described earlier. ${ }^{19}$ All reagents were prewarmed to $37^{\circ} \mathrm{C}$ before use. $400 \mu \mathrm{l}$ of neutrophil suspension was mixed with $600 \mu \mathrm{l}$ luminol solution and $200 \mu \mathrm{l}$ crystal suspension and immediately placed in an LKB luminometer (model 1250). Light output in millivolts was recorded on a chart recorder and digital $\stackrel{\infty}{\oplus}$ printout set to 10 -second recording integrals. 음 Temperature was kept at $37^{\circ} \mathrm{C}$ by a water jacketed sample holder.

PREINCUBATION WITH MRF IgG-coated and uncoated MSU (10 g/l) were preincubated with a $1 \% \mathrm{v} / \mathrm{v}$ solution of purified

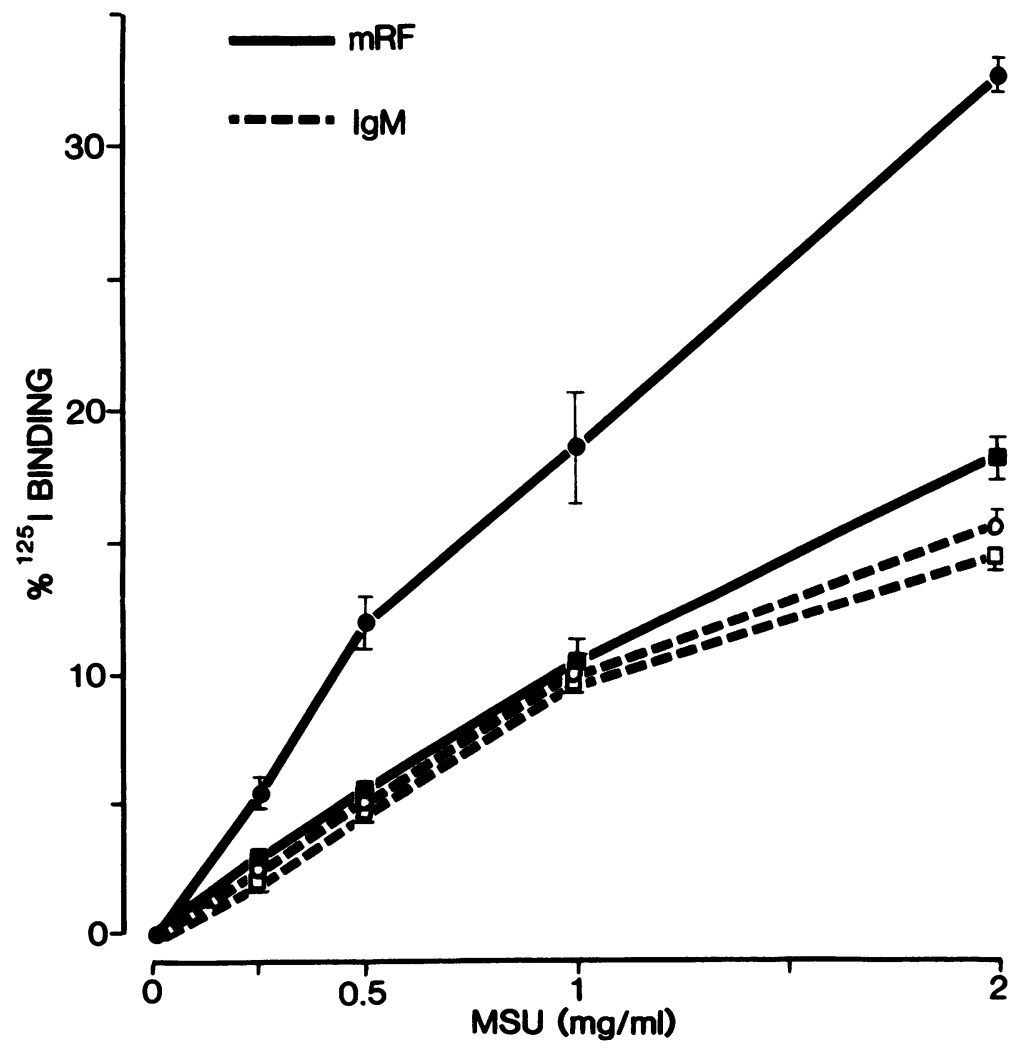

Fig. 2 Binding of trace amounts of 125I-labelled $m R F$ and IgM to uncoated MSU (squares) and IgG-coated MSU (circles). Mean $\pm S D ; n=3$. 

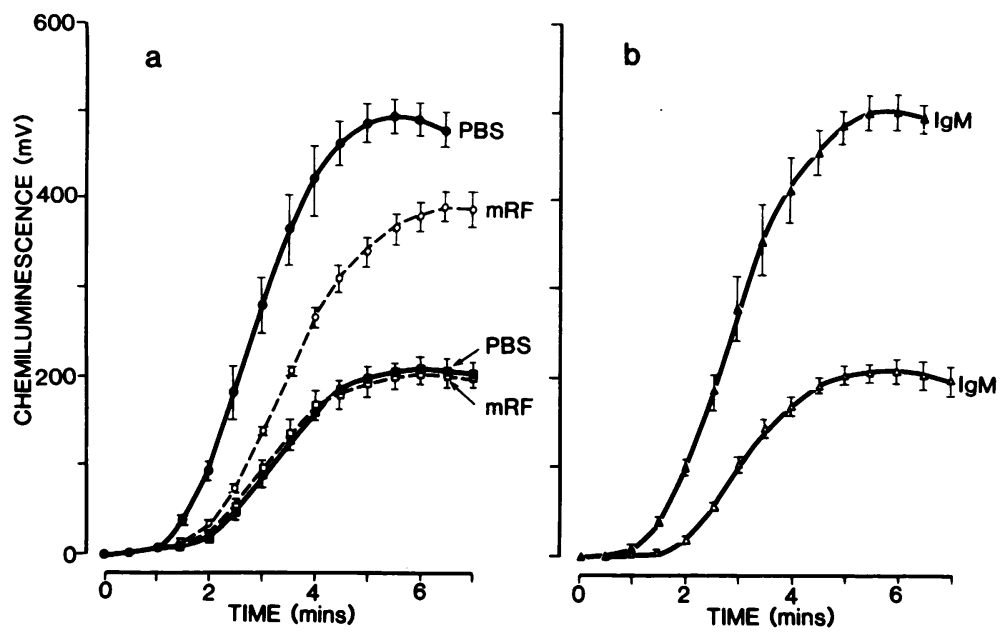

Fig. 3 The initial CL responses of $2 \times 10^{6}$ neutrophils on addition of $600 \mu \mathrm{l}$ luminol and $200 \mu \mathrm{l} \mathrm{MSU}$ suspension. (a) The solid lines indicate the $C L$ response to uncoated MSU (ם) and IgG-coated MSU (O) both preincubated with $P B S$. The dashed lines indicate the $C L$ responses after incubation of uncoated MSU ( $\square)$ and IgG-coated $M S U(O)$ with $M R F$. (b) Activation of neutrophils by uncoated $M S U(\triangle)$ and IgG-coated $M S U(\mathbf{\Delta})$ both preincubated with an IgM control. Mean $\pm S D ; n=4$.

$\mathrm{mRF}(0.5 \mathrm{~g} / \mathrm{l})$ and $\mathrm{IgM}$ control $(0.5 \mathrm{~g} / \mathrm{l})$ for 60 minutes at room temperature and washed before testing for neutrophil activation by CL. Control crystals were preincubated with PBS alone.

\section{Results}

BINDING OF MRF AND IgM TO MSU Precoating of MSU with human IgG enhanced the binding of mRF by approximately twofold over a range of crystal concentrations, whereas IgM bound with similar affinity to both crystal preparations (Fig. 2). This suggests that the mRF can bind to the crystal surface via previously adsorbed IgG.

EFFECT OF BINDING OF MRF ANDIgM TO MSU ON CL

The possible functional consequences of $\mathrm{mRF}$ and IgM binding on crystal-neutrophil interactions were studied by a CL assay. The CL response was enhanced two- to threefold by coating the crystal with IgG (Fig. 3a), confirming the observation of Platt et al. ${ }^{26}$ Preincubation with mRF $(1 \% \mathrm{v} / \mathrm{v}$ of a $0.5 \mathrm{~g} / \mathrm{l}$ solution) significantly reduced the maximum CL response with IgG-coated MSU $(p<0.01$; twotailed Student's $t$ test) without inhibiting the response with uncoated crystals (Fig. 3a). The same concentration of the IgM control had no effect on uncoated or IgG-coated MSU (Fig. 3b).

\section{Discussion}

Our patient is an example of one of the few convincing cases of coexistent gout and RA and only the fourth case of gout with documented MSU and RA with a rheumatoid nodule. Some reports of supposed coexistence can be viewed critically, since the diagnosis of RA was based on the presence of morning stiffness, symmetrical synovitis, and a positive RF titre, features that can be mimicked by polyarticular gout. ${ }^{27-29}$ We cannot explain the association between the two diseases in our patient. In particular there appeared to be no correlation between the degree of hyperuricaemia, the RF titre, and the clinical expresson of his gout and RA.

The pathogenesis of gouty inflammation is complex and not fully understood, but the interaction between MSU and neutrophils appears central. ${ }^{30}$ Binding isotherms of $\mathrm{IgG}$ to urate crystals have suggested monomolecular adsorption at concentrations of IgG found in joint fluid, and studies with molecular probes have shown that the Fc fragments of the IgG molecules are fully exposed. ${ }^{31} 32$ Bardin et al. ${ }^{33}$ have confirmed the functional availability of the Fc fragment by immunoelectron microscopy. Crystals coated with IgG have been shown to enhance non-cytolytic secretion of lysosomal enzymes and superoxide anion, presumably due to interaction of crystal-bound IgG with Fc receptors on the neutrophil membrane. ${ }^{21} 34$ Thus IgG crystal coating may have an important role in triggering acute gouty arthritis. RF binds to epitopes on the Fc portion of $\mathrm{IgG} .{ }^{35}$ We propose that adsorption of IgG to the crystal surface increases the density of Fc antigenic determinants, favouring multivalent binding of RF. This is supported by the enhanced binding of $\mathrm{mRF}$ to IgG-coated MSU (Fig. 2). Similarly the preferential reactivity of $\mathrm{RF}$ with aggregated or complexed IgG is possibly due to the increased number of exposed IgG Fc antigenic determinants in the complexed state. ${ }^{22} \mathrm{We}$ postulate a masking of the specific IgG $\mathrm{Fc} / \mathrm{Fc}$ receptor 
interaction by mRF. This is suggested by our findings that $\mathrm{mRF}$ inhibited neutrophil activation by IgG-coated MSU in a dose that had no effect on uncoated crystals (Fig. 3a). In contrast, coating with the IgM control did not alter the response to either crystal preparation (Fig. 3b). We have shown a similar inhibition by $\mathrm{mRF}$ of neutrophil activation by large immune complexes (IC), which raises the speculation that IgG-coated urate crystals in vivo may have some properties similar to IC. ${ }^{19}$

The present in-vitro studies suggest a role for RF in modulation of crystal-neutrophil interactions mediated by adsorbed IgG. The effect of RF on MSU activation of other cellular and humoral mediators of inflammation requires further study. Whether RF plays a significant role in modifying gouty arthritis in vivo is not clear. With a neutrophil monolayer system to study neutrophil responses to urate crystals Turner et al. ${ }^{18}$ reported no effect of RF on adherence, phagocytosis, or enzyme release, but the RF used was polyclonal and unpurified. Doherty et al. ${ }^{36}$ showed that patients with seropositive RA mount normal inflammatory responses to intradermal injection of MSU, but this model of crystal-induced inflammation may not allow participation of RF during induction of the inflammatory response. It seems unlikely that crystal coating by RF alone can explain the mutual exclusion of gout and RA. For example, RF would not be expected to prevent the development of tophaceous deposits. A combination of genetic, biochemical, and immunological factors may account for the apparent dissociation between these two diseases.

We gratefully thank $\mathrm{Dr}$ S Neoh and Mr T Nikoloutsopoulos for technical assistance and Ms L Koh for purifying the proteins. T P Gordon was supported by an $\mathrm{NH}$ and MRC postgraduate scholarship.

\section{References}

1 Wallace D J, Klinenberg J R, Morhaim D, Berlanstein B, Biren P C, Callis G. Coexistent gout and rheumatoid arthritis. Case report and literature review. Arthritis Rheum 1979; 22: 81-6.

2 Trentham D E, Masi A T. Chronic synovitis in gout simulating rheumatoid arthritis. Demonstration of bilateral popliteal cysts and wrist synovial corrugation. JAMA 1976; 235: 1358-60.

3 Talbott J H, Altman R D, Yu T F. Gouty arthritis masquerading as rheumatoid arthritis or vice versa. Semin Arthritis Rheum 1978; 8: 77-114.

4 Kozin F, McCarty D J. Rheumatoid factors in the serum of gouty patients. Arthritis Rheum 1977; 20: 1559-60.

5 Rappoport A S, Susman J L, Weissman B N. Lesions resembling gout in patients with rheumatoid arthritis. Am J Roentgenol 1976; 126: 41-5.

6 Goldenberg D L, Cohen A S. Synovial membrane histopathology in the differential diagnosis of rheumatoid arthritis, gout, pseudogout, systemic lupus erythematosus, infectious arthritis and degenerative joint disease. Medicine (Baltimore) 1978; 57: 239-52.
7 McCarty D J. The pendulum of progress in gout with crystals to $\frac{\square}{\omega}$. hyperuricaemia and back. Arthritis Rheum 1964; 7: 534-41.

8 Owen D S. Toone E. Irby R. Coexistent rheumatoid arthritis $\overrightarrow{\bar{F}}$ and chronic tophaceous gout. JAMA 1966; 197: 123-6.

9 Jesee E F, Toone E, Owen D S, Irby R. Coexistent rheumatoid arthritis and chronic tophaceous gout. Arthritis Rheum 1980; 23: 244-7.

10 Agudelo C A, Turner R A, Panetti M, Pisko E. Does $\frac{\sqrt{D}}{\overparen{D}}$ hyperuricaemia protect from rheumatoid inflammation? Arthri- @ tis Rheum 1984; 27: 443-8.

11 Lussier A, DeMedicis R. Inhibition of adjuvant-induced 2 arthritis in the hyperuricaemic rat. Agents Actions 1978; 8: $\vec{\circ}$ 536-42.

12 Schwartz M L, Malawista S E. Dosage-dependent inhibition of $\vec{\omega}$ phagocytosis by human leukocytes exposed to urate in solution (abstract) Arthritis Rheum 1975; 18: 424

13 Turner R A, Pisko E J, Agudelo C A, Counts G B, Foster S L. ?ำ Uric acid effects on in vitro models of rheumatoid inflammatory $\vec{A}$ and autoimmune processes. Ann Rheum Dis 1983; 42: 338-42. ஜூ

14 Ames B N, Cathcart R, Schwiers E, Hochstein P. Uric acid $\omega$ provides an antioxidant defense in humans against oxidant- and $\underset{\oplus}{\infty}$ radical-caused aging and cancer: a hypothesis. Proc Natl Acad 0 Sci USA 1981; 78: 6858-62.

15 Schumacher H R. Pathogenesis of crystal-induced synovitis. Clin Rheum Dis 1977; 3: 105-31.

16 Scott J T. Gout. Ann Rheum Dis 1983; 42 (suppl): 16-8.

17 Turner R A, Schumacher H R, Myers A R. Phagocytic function of polymorphonuclear leucocytes in rheumatic diseases. J Clin $\vec{\emptyset}$ Invest 1973; 52: 1632-5.

18 Turner R A, Counts G B, Treadway W J, Holt D A, Agudelo C A. Rheumatoid factor and monosodium urate crystalneutrophil interactions in gouty inflammation. Inflammation 1981; 5: 353-61.

19 Gale R, Bertouch J V, Gordon T P, Bradley J, RobertsThomson P J. Neutrophil activation by immune complexes and $ᄋ$ the role of rheumatoid factor. Ann Rheum Dis 1984; 43: 34-9.

20 Denko C W, Whitehouse $\mathrm{M}$ W. Experimental inflammation induced by naturally occurring microcrystalline calcium salts. $J$ 윽 Rheumatol 1976; 3: 54-62.

21 Abramson S, Hoffstein S T, Weissman G. Superoxide anion generation by human neutrophils exposed to monosodium urate. Effect of protein adsorption and complement activation. Arthritis Rheum 1982; 25: 174-80.

22 Roberts-Thomson $\mathrm{P}$ J. A reappraisal of the monoclonal rheumatoid factor test for circulating immune complexcs: a comparison of two monoclonal rheumatoid factor reagents. Clin Exp Immunol 1982; 48: 52-62.

23 Hesser C, Boesman M, Nordin J H, Isliker H. Effect of chemical and enzymatic radioiodination on in vitro human $\mathrm{Clq}$ 음 activities. J Immunol 1973; 110: 820-8.

24 Terkeltaub R, Curtiss L K, Tenner A J, Ginsberg M H. Lipoproteins containing apoprotein $\mathrm{B}$ are a major regulator of neutrophil responses to monosodium urate crystals. J Clin $\mathrm{N}$ Invest 1984; 73: 1719-30.

25 Allen R C, Stjernholm R L, Steele R H. Evidence for the $N$ generation of an electronic excitation state(s) in human poly-N morphonuclear leukocytes and its participation in bactericidal $\omega$

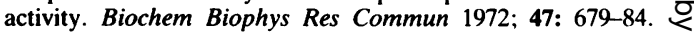

26 Platt P N, Jeffery S, Wilson I D, Griffith I D. The interactionso of crystals and polymorphonuclear leukocytes monitored by luminol dependent chemiluminescence. Ann Rheum Dis 1981 : S 40: 615 .

27 Schwartzberg M, Lieberman D H, Gupta V P, Ehrlich G E. Rheumatoid arthritis and chronic gouty arthropathy. JAMA 1978; 240: 2658-9.

28 Rizzoli A J, Trujeque L, Bankhurst A D. The coexistence of $\frac{\vec{D}}{2}$ gout and rheumatoid arthritis: case report and a review of the $\frac{\varrho}{\sigma}$
literature. J Rheumatol 1980; 7: 316-24.

29 Pistiner M, Pitlik S, Rosenfeld J B. Gout, pseudogout and $\Omega$ 
rheumatoid arthritis in an elderly patient. Med Interne 1982; 20: 55-8.

30 Phelps P, McCarty D J. Crystal-induced inflammation in canine joints. II. Importance of polymorphonculear leucocytes. J Exp Med 1966; 124: 115-25.

31 Kozin F, McCarty D J. Protein binding to monosodium urate monohydrate, calcium pyrophosphate dihydrate and silicon dioxide crystals. I. Physical characteristics. J Lab Clin Med 1977; 89: 1314-25.

32 Kozin F, McCarty D J. Molecular orientation of immunoglobulin $\mathrm{G}$ adsorbed to microcrystalline monosodium urate monohydrate. J Lab Clin Med 1980; 95: 49-58.
33 Bardin T, Cherian P V. Schumacher H R. Immunoglobulins on the surface of monosodium urate crystals: an immunoelectron microscopic study. J Rheumatol 1984; 11: 339-41.

34 Kozin F. Ginsberg M. Skosey J. Polymonophonuclear leukocyte responses to monosodium urate crystals: modification by adsorbed serum proteins. J Rheumatol 1979; 6: 519-26.

35 Johnson P M. Molecular nature and cross-reactions of rheumatoid factor. In: Holborow E J, ed. Clinics in immunology and allergy. London: Saunders, 1981: 103-15.

36 Doherty M, Hornby J, Dieppe P A. Normal response to monosodium urate crystals by patients with rheumatoid arthritis. Ann Rheum Dis 1983; 42 (suppl): 95-6. 